\title{
A device mimicking the biomechanical characteristics of crocodile skull for lumbar fracture reduction
}

Jingmin $\mathrm{Li}^{1}$, Lihua $\mathrm{Guo}^{1}$,Yuancheng $\mathrm{Li}^{2}$, Zhenkun $\mathrm{Lei}^{3}$, Yuanchang $\mathrm{Liu}^{4}$, Weiping $\mathrm{Shi}^{1}$, Tao $\mathrm{Li}^{1}$, Weikang $\mathrm{Li}^{3}$ and Chong $\mathrm{Liu}^{1,5^{*}}$

${ }^{1}$ Key Laboratory for Micro/Nano Technology and System of Liaoning Province, Dalian University of Technology, Dalian, China,116024.

${ }^{2}$ Department of Orthopedics, First Affiliated Hospital of Dalian Medical University, Dalian, China, 116024.

${ }^{3}$ State Key Laboratory of Structural Analysis for Industrial Equipment, Dalian University of Technology, Dalian, China, 116024.

${ }^{4}$ Department of Mechanical Engineering, University College London, London, UK, NW1 2BX

${ }^{5}$ Key Laboratory for Precision and Non-traditional Machining Technology of Ministry of Education, Dalian University of Technology, Dalian, China,116024.

Email: jingminl@dlut.edu.cn; chongl@dlut.edu.cn

\begin{abstract}
Open surgery is currently the main treatment method for the lumbar burst fracture with neurological deficit but may irreversibly disrupt the lumbar anatomy. The minimally invasive surgery (MIS) techniques have gained increasing attentions recently. However, their use is still limited to lumbar burst fractures mainly due to their difficulties in burst fracture reduction and decompression. Here we present a novel bio-inspired MIS device which can be used with an endoscope to reset the bone fragments retropulsed into the spinal canal within the wounded vertebral body. Its head jaw mimics the biomechanical characteristics of a crocodile rostrum to improve the performance in gripping and moving bone pieces in the confined space of a vertebral body. This study may be capable of converting the posterior open surgeries to the MIS procedures, and expands the use of the MIS techniques in the treatment of lumbar burst fractures.
\end{abstract}

\section{Introduction}

The lumbar burst fracture can be caused by a high-energy axial load making the bone fragments of a vertebra section penetrate surrounding tissues and sometimes the spinal canal ${ }^{1}$. A burst fracture without neurologic injury may be treated with nonsurgical method, such as the Thoracic Lumbar Sacral Orthosis (TLSO) or the body cast ${ }^{2,3}$. On the other hand, surgical 
treatment is required when the fracture results in a spinal cord injury.

During the surgical treatment, fracture reduction and spinal canal decompression are usually necessary ${ }^{4-7}$. In the anterior and posterior surgeries which are commonly used for spinal burst fractures, they are always achieved by removing portions of the injured vertebra, discs and surrounding tissues. This surgical procedure has many disadvantages, including the irreversible disruption of lumbar anatomy, the damage to muscles as well as tissues and the significant operative blood loss ${ }^{8-10}$. The minimally invasive surgery (MIS) techniques, such as percutaneous kyphoplasty (PKP), percutaneous vertebroplasty (PVP) and spinal fusion ${ }^{11-14}$, have gained interest in the past two decades. The complement of minimally invasive percutaneous posterior lumbar interbody fusion (MIP-PLIF) ${ }^{15}$ and minimally invasive surgery transforaminal lumbar interbody fusion (MIS-TLIF) ${ }^{16}$ have made a breakthrough in the treatment of spinal fractures. Some MIS devices, like "Spine Assist" system, SPINEBOT ${ }^{17}$ and CoRass ${ }^{18}$, have emerged recently. However, they may be still contraindicated for the treatment of lumbar burst fractures due to their limitations in resetting the bone fragments retropulsed into the spinal canal and achieving decompression. Thus, a new MIS device, which can overcome these disadvantages, is critical.

In this work, we present a novel bio-inspired MIS device, which has the potentials to become the auxiliary tool for fracture reduction in posterior surgeries. By being percutaneously put into the vertebral body through a pedicle canal, it is able to effectively pull the bone fragments invading into the spinal canal back to their initial positions (figure 1a).

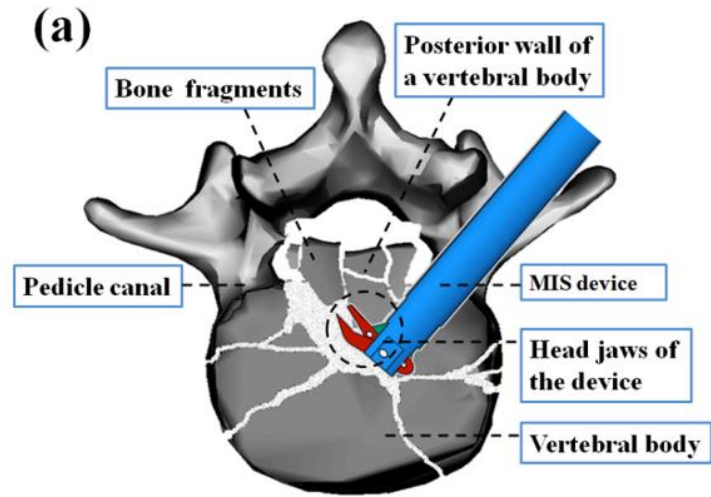

A MIS device used to rebuild lumbar vertebral body

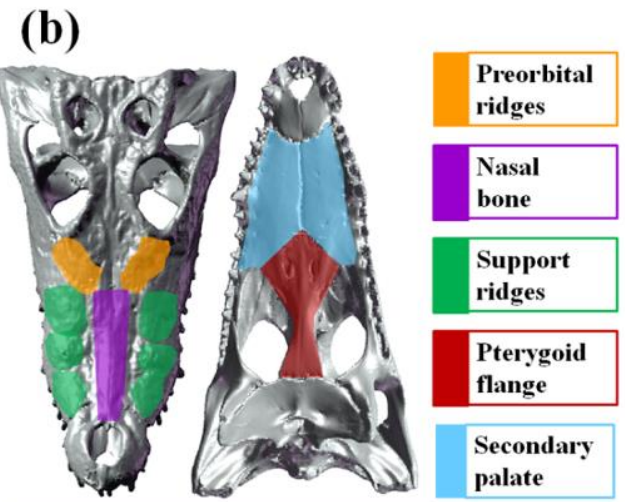

Strain-reducing structures on a crocodile skull

(c)

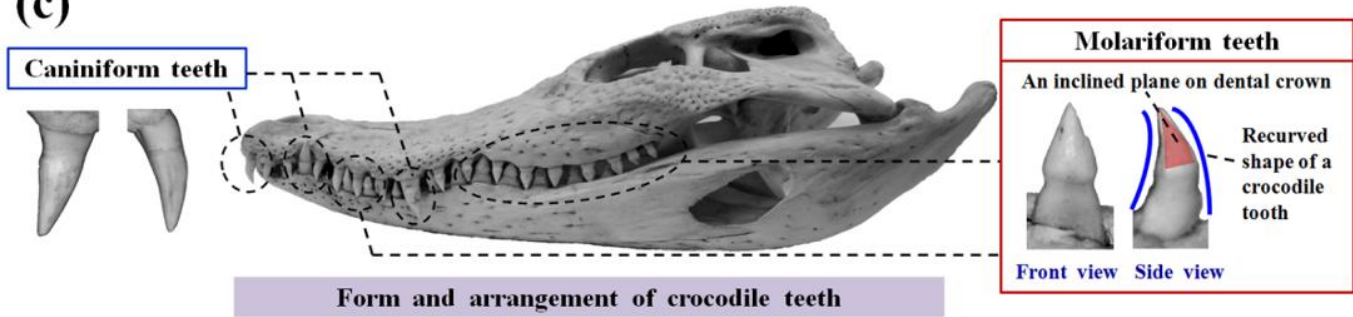

Figure 1. A novel minimally invasive surgery device for vertebral burst fracture reduction and the biomechanical characters of crocodilian rostrum and molariform tooth. (a) The MIS device can be put into the vertebral body through a pedicle canal to reset the vertebral fragments. (b) The strain-reducing features on a crocodilian skull. (c) The molariform teeth with recurved shape and an inclined plane on the dental crown, which are useful in gripping preys.

When developing the device, there is a trade-off between the size and the mechanical 
stiffness of the device. In order to be fitted into an approximate $6 \mathrm{~mm}$ pedicle canal and operating in the confined space of a wounded vertebra (about $3 \sim 4 \mathrm{~cm}^{3}$ for an adult), the head jaws of the device must be small and slim. However, the slim head jaws with low mechanical stiffness have difficulties in firmly gripping and moving the vertebral fragments covered by slippery marrow. Therefore, it is important to improve the mechanical performance and gripping firmness of the head jaws while keeping the device size compact.

The structure of the crocodile rostrum has provided such a performance. Crocodiles belong to apex predators with platy and long rostral skulls and are able to resist load up to several hundreds or even thousands kilograms ${ }^{19,20}$. It has been found that various strain-reducing features, such as the pterygoid flange, the preorbital ridges, the nasal bone, as well as the longitudinal and transverse support ridges on the maxilla (figure 1b), can indicate the major load pathway within the rostrum, relief the increased mechanical strength and eliminate stress concentration at local region ${ }^{21-23}$. With these features, crocodilian rostrum can resist large loads without increasing bone size or thickness. In addition, the arrangement and the dental form of the molariform teeth within the crocodilian rostrum are helpful in gripping prey $^{24-27}$. Crocodilian molariform teeth are located in the posterior rows of the rostrum to crush prey and hold them firmly. Each molariform tooth has curved shape with an inclined plane on the dental crown (figure 1c). These features can enhance the structural rigidity as well as thearea moment of inertia and reduce the bending moments. The unique structure of the crocodile rostrum has inspired us to design the head jaws of the MIS device. First, by mimicking the strain-reducing features of crocodilian rostrum, the mechanical performance of the head jaws can be increased evidently without enlarging their sizes. Second, the microstructures mimicking the molariform teeth distribution and dental shapes can be fabricated on the occlusal surfaces of the head jaws to improve the gripping firmness of bone pieces.

\section{Methods}

\subsection{Ethical statement}

All experimental protocols were approved by the department of Orthopedics, First Affiliated Hospital of Chinese Dalian Medical University. The human lumbar used in the experiment was provided by the department of Orthopedics, First Affiliated Hospital of Chinese Dalian Medical University. All experiments conducted in this research were performed in accordance with the institutional guidelines and management of research projects in Dalian Medical University.

\subsection{The design of the head jaws}

The 3D model of an adult Siamensis crocodile skull was built from CT scan data based on the Reverse-Engineering (RE) method ${ }^{28}$. The sizes of various strain-reducing features in the rostrum were obtained from the 3D model (see table 1). The width and thickness across the posterior preorbital ridge borders are defined as the rostrum width and rostrum thickness, respectively ${ }^{29,30}$. The nasal bone (purple colour in figure 1a) is adjacent to two preorbital ridges (orange colour). They form a Y-shape support structure. The average width of the nasal bone is approximate $1 / 5$ the width of the rostrum, and its height is approximate 1/15-1/12 the 
thickness of the rostrum. The total width of two preorbital ridges is nearly $1 / 3-1 / 2$ the width of the rostrum. The height of a preorbital ridge is about $1 / 8$ the thickness of the rostrum. The support ridges (green colour in figure 1a) include the longitudinal ridges at the rostrum surface and the circular ridges around the nostril. In essence, each ridge composes of several sculpturing characteristic protrusions whose heights are 1/14-1/12 the thickness of the rostrum. The widths of the protrusions are 1/6-1/5 the width of the rostrum. The pterygoid flange (red colour in figure 1a), which has the arched shape, connects the upper palate of the rostrum with the occipital bone. Its width is $1 / 3-1 / 2$ the width of rostrum. The average bone thickness of the pterygoid flange is about $1 / 11$ the thickness of the rostrum.

Table 1. The sizes of various strain-reducing features in a crocodile rostrum.

\begin{tabular}{ccccc}
\hline Sizes & Nasal bone & Preorbital ridge & Support ridge & Pterygoid flange \\
\hline Width $(\mathrm{mm})$ & 16.5 & $33.4(16.7 \times 2)$ & $15.0-17.1$ & $25.4-39.2$ \\
Height $(\mathrm{mm})$ & $2.0-2.4$ & 3.7 & $2.2-2.5$ & 2.7 \\
\hline
\end{tabular}

${ }^{a}$ The width and the height of the crocodile rostrum are $82.6 \mathrm{~mm}$ and $29.8 \mathrm{~mm}$, respectively.

${ }^{\mathrm{b}}$ The width of a preorbital ridge is $16.7 \mathrm{~mm}$. The sum of two preorbital ridges is $33.4 \mathrm{~mm}$.

${ }^{\mathrm{c}}$ Nasal bone width, preorbital ridge width and height, as well as pterygoid flange height are all the average of a series of measurement values.

The upper and the lower head jaws of the device both have been designed by mimicking the above-mentioned strain-reducing features. The specific dimensions of the bio-inspired structures on the jaws were designed by mimicking the size ratios of the strain-reducing structures to the crocodile rostrum. Furthermore, all the sizes of the biomimetic structures on the jaws have also been optimized by using FEA method. The optimizing process can be seen in "Supplementary data-2. The size optimization of the bio-inspired structures".

Figure 2a shows the structure of the upper jaw. According to the definition of crocodilian rostrum width and thickness, the width across the posterior borders of the yellow area (which has been designed to mimic the preorbital ridges) is defined as the jaw width (which is $4 \mathrm{~mm}$ ), and the thickness of the jaw at this position is defined as the jaw thickness (which is $2.6 \mathrm{~mm}$ ). The microprotrusions with green colour are used to mimic the support ridges on crocodile rostrum. The heights of the microprotrusions are all $200 \mu \mathrm{m}$ which is about $1 / 13$ the thickness of the jaw. The average width of each microprotrusion has been designed as $0.8 \mathrm{~mm}$ which is about 1/5 the width of the jaw. The longitudinal beam highlighted with orange colour in the middle of the jaw is used to mimic the nasal bone of the crocodile rostrum. Its width and height are $800 \mu \mathrm{m}$ and $200 \mu \mathrm{m}$ which are $1 / 5$ and $1 / 13$ the width and the thickness of the jaw respectively. The arched protrusions highlighted with yellow colour are designed to mimic the preorbital ridges. They increase approximate 1/6 the average thickness of the grasping part. The total width of two arched protrusions is about $1 / 2$ the width of the jaw. The red colour structure is used to mimic the pterygoid flange. It has an arched shape and connects the front part of the jaw with the back support structures. Its width is $1 / 2$ the width of the jaw, and its thickness is $220 \mu \mathrm{m}$ which is about $1 / 11$ the average thickness of the jaw.

\subsection{Microstructures on the occlusal surfaces of the jaws}

Based on the characteristics of the molariform teeth in a crocodilian rostrum, an array of microstructures which mimic the forms of the molariform teeth have been fabricated on the occlusal surfaces of the upper and the lower jaws of the MIS device (figure 2b). Each 
microstructure was designed to be pyramid-like with an inclined plane at its top. The angle of the inclined plane is approximately $22^{\circ}$. Also, each pyramid-like structure is recurved with a curvature radius of $1.12 \mathrm{~mm}$.

To enhance the contact area with an irregular-shape bone, the microstructures have been designed with two different sizes, i.e. the large structure with the height of $300 \mu \mathrm{m}$ and the square base having a side length of $400 \mu \mathrm{m}$, the small structure with the height of $200 \mu \mathrm{m}$ and the rectangle base having a length of $400 \mu \mathrm{m}$ and a width of $300 \mu \mathrm{m}$. The large structures are located at the middle and the anterior of the jaw and below the support ridges (green colour area in figure 2a), which are well suited for ensuring enough gripping pressure. On the contrary, the small ones are located near the large structures, which can be auxiliary in grasping bone fragments.

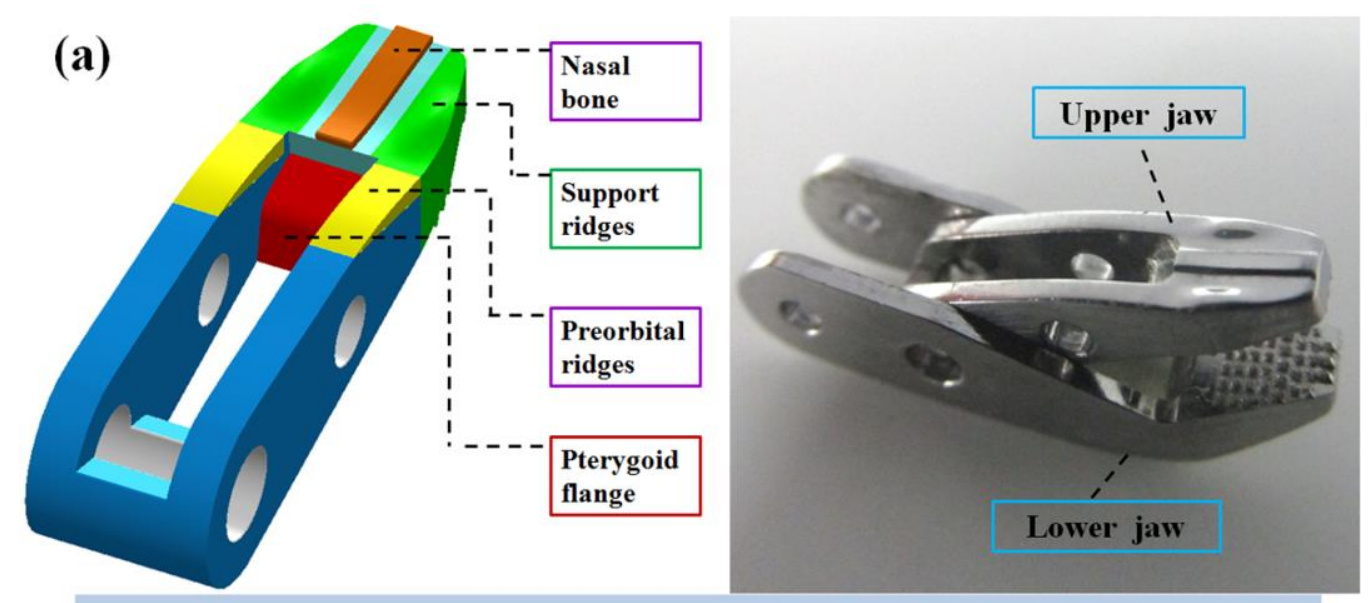

The jaws mimics the strain-reducing features of the crocodile rostrume

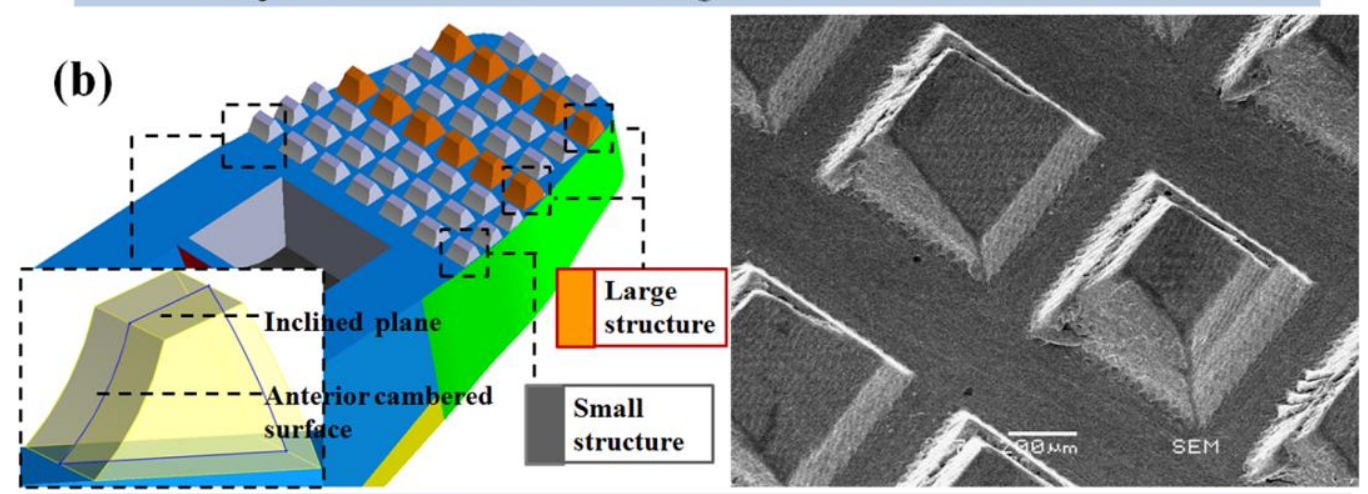

The microstructures on the occlusal surfaces of the jaw mimic crocodile dental forms

Figure 2. Design of the jaws by mimicking crocodilian strain-reducing features and dental forms. (a) The jaws designed by mimicking the strain-reducing features of a crocodilian skull to increase their bending stiffness. (b) The microstructures on the occlusal surface of a jaw designed by mimicking the dental form of a crocodilian tooth to enhance gripping performance to the bone pieces.

The distribution of the microstructures was also designed according to the crocodile teeth. The microstructures close to the joint of the grasper are shorter (figure 2b), which are consistent with the structure found in crocodilian teeth (figure 1c). Such a design can decrease the concentration of pressure at the area close to the joint of the grasper and equalize the pressure across the length of the jaw. The interval between two adjacent microstructures is $200 \mu \mathrm{m}$. All these teeth distributing on the upper and lower graspers can occlude without 
interference.

\subsection{Measurement of the mechanical deformation of the jaws}

Two stainless steel jaws were fabricated for testing with one having the bio-inspired strain-reducing microstructures and the other one not. The deformations of these two jaws were recorded by using the digital image correlation (DIC) method. In order to satisfy the requirements of the DIC, the specimens were enlarged by three times from their original size. A thin layer of white paint was sprayed on the lateral side of each specimen followed by an overspray of black paint to obtain a random speckle pattern. The specimens were mounted onto a universal testing machine (Instron 3345R2773, Instron Corporation, Canton, MA, USA) which can continuously control the load applied onto the specimens. Two cameras (Computar 35mm 1:1.4 2/3, Computar Company, Japan) connecting to an image acquisition system were placed $1.0 \mathrm{~m}$ from the specimen and were aligned to be approximately perpendicular to the side surface of the specimen. During the test, a load of $800 \mathrm{~N}$ with a loading rate of $160 \mathrm{~N} / \mathrm{min}$ was applied onto the front-end of the specimen to make it bending. The deformation images were recorded at a rate of 2 frames per second. All the images obtained were imported into specialized DIC software for processing, and the specimen deformation and strain can be obtained.

\subsection{The measurement of gripping performance}

Crocodile-tooth-like microstructures, triangular serratures and wavelike serratures were fabricated on three pairs of 400 stainless metal plates respectively (see Supplementary data-5. Measurement of gripping performance). Each plate has the dimension of $50 \mathrm{~mm} \times 16 \mathrm{~mm}$. All of the fabricated microstructures had the same size $(200 \mu \mathrm{m}$ high, $600 \mu \mathrm{m}$ wide and $120 \mu \mathrm{m}$ line spacing).A pair of plates with the same serrated surfaces was mounted onto a universal testing machine (WDW-300E, Jinan Shijin Group Corporation, China). A fresh bone of $10 \mathrm{~mm} \times 6 \mathrm{~mm} \times 5 \mathrm{~mm}$ cut from a porcine vertebra was put between them, and the bone surface has been milled to be flat enough. A compression force of $15 \mathrm{~N}$ to $30 \mathrm{~N}$ was applied. A digital push and pull tester was used to pull the bone sliding between the two serrated surfaces of the plates. The compression force and the pull force were recorded in real-time by a computer. It can be known that the pull force was two folds of the friction force. The friction coefficient can be calculated by equation $\mu=\mathrm{F} / \mathrm{P}$ (where $\mathrm{F}$ is the friction force which is half of the pull force, $\mathrm{P}$ is the compression force). The experiment was repeated three times for each kind of serrated surface (each experiment used a new bone) with the friction coefficient been chosen as the average of three experimental values.

In the measurement experiments of the sliding coefficient, the bone specimens were also cut from the porcine vertebras but without surface milling to keep their original uneven surfaces. The experimental procedure was similar to the friction coefficient's. As the bone just began to slide between the two serrated surfaces of the upper and the lower plates, the pull force, which is named as the sliding force, was recorded. By changing the compression forces, a series of sliding forces can be obtained, and a relationship graph between the sliding force and the compression force can be established. The graph was always called the slip-line, the slope of which was the sliding coefficient. 


\subsection{Establishing of the burst fractured vertebral models}

Three calve vertebrae with intervertebral discs were bought from market. Their soft tissue was carefully cleaned off. However, the ligaments and in tervertebral discs were retained. Pre-damage ${ }^{35,36}$ was made at the middle section of the vertebra by drilling several holes into its posterior wall (the wall adjacent to spinal nerves). The top and bottom vertebrae were encased in the denture base resins to level the top and the bottom plane of the specimen. A universal testing machine (WDW-300E, Jinan Shijin Group Corporation, China) was used to create the experimental burst fractures. First, an axial constant compression force with $1 \mathrm{kN} / \mathrm{s}$ rate was applied on the specimen until the burst fracture was formed. After removing the top and bottom vertebrae, a burst fractured vertebral model was obtained. Then, a human lumbar of L4 segment was used to make the burst fractured vertebral model. To form a man-made nervous cord, Polydimethylsiloxane (PDMS) with a small amount of wires was inserted into the spinal canal of the lumbar. Then the lumbar was compressed by using the process mentioned above to generate the burst fractures (see Methods) on its posterior wall. The experiments were carried out in accordance with the guidelines issued by the Ethical Committee of Dalian University of Technology.

\section{Results and Discussion}

\subsection{Biomechanical analysis of a crocodile skull}

To evaluate the mechanical performance of crocodile skull, we performed a biomechanical analysis by using the finite element analysis (FEA) in this paper.
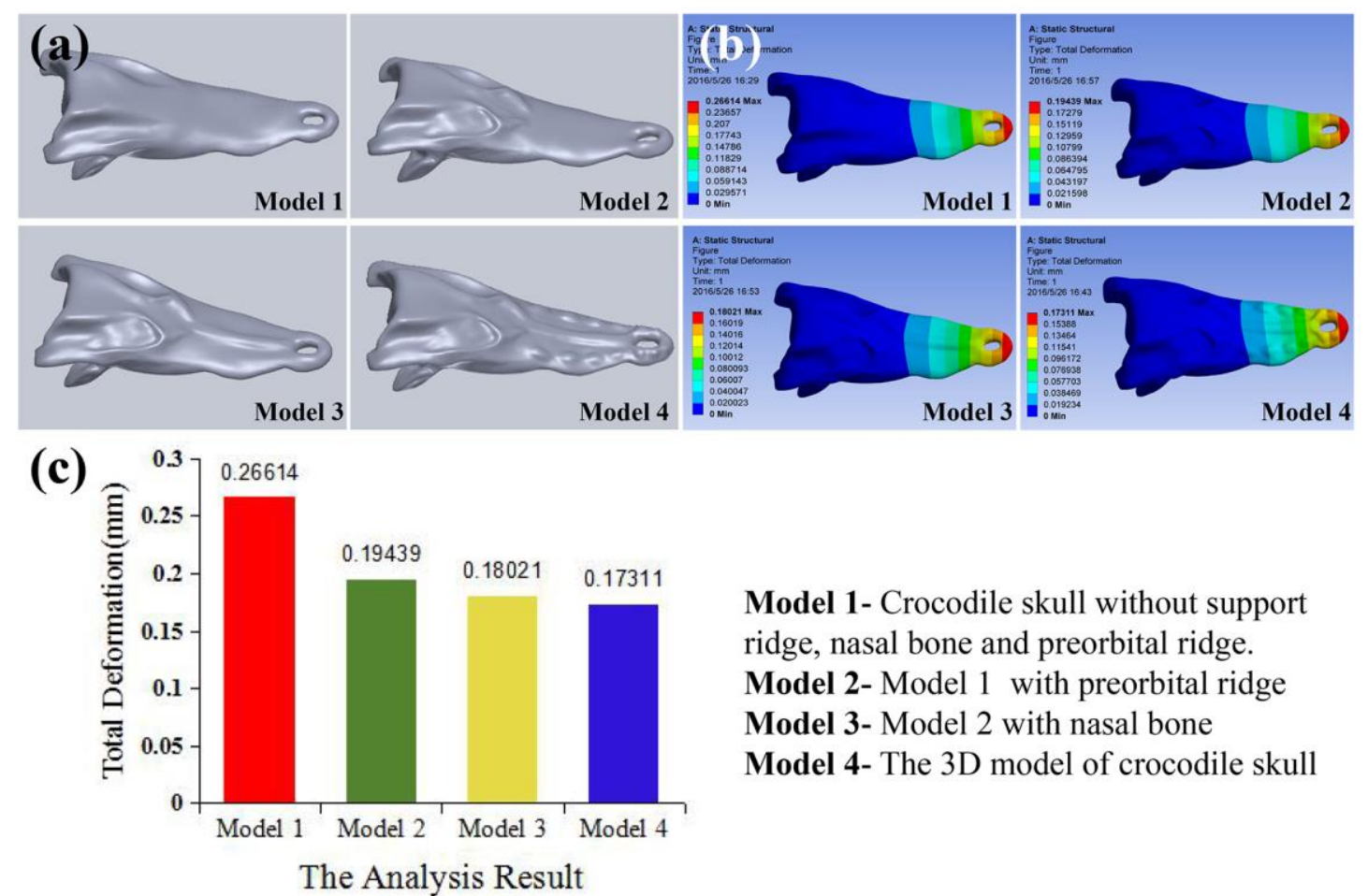

Model 1- Crocodile skull without support ridge, nasal bone and preorbital ridge. Model 2- Model 1 with preorbital ridge Model 3- Model 2 with nasal bone Model 4- The 3D model of crocodile skull

Figure 3. Finite element analysis of different models to contrast their mechanical performance. (a) Contrast models without the strain-reducing structures and a 3D model of an adult Siamensis crocodile skull used for FEA. (b) ANSYS analysis of these models. (c) The analysis results. 
A series of contrast models have been built by using the Reverse-Engineering (RE) method (see Supplementary data-1. 3D model of a Siamese crocodile skull). As shown in figure $3 \mathrm{a}$, model 1 is a skull without support ridges, nasal bone and preorbital ridges. Model 2 is built by adding the preorbital ridges to model 1 . Model 3 is built by adding nasal bone to model 2. Model 4 is the complete crocodile skull with all strain-reducing features. Finite element simulations (figure $3 \mathrm{~b}$ ) are performed to analyze the deformation of these models. The bending forces being applied onto the models are all 3000N.

The analysis results have been shown in figure $3 \mathrm{c}$. It has been found that the maximum bending deformations of model 1 , model 2, model 3 and model 4 are approximately $266.14 \mu \mathrm{m}, 194.39 \mu \mathrm{m}, 180.21 \mu \mathrm{m}$ and $173.11 \mu \mathrm{m}$, respectively. Results show that the bending deformation has obviously decreased as the strain-reducing features are added to the analysis model. As being compared to a skull without the strain-reducing features (model 1), the deformation of a complete crocodile skull (model 4) will reduce $34.96 \%$ at same load conditions. This analysis can exhibit that the strain-reducing features can increase the robustness against deformation in a crocodile skull.

\subsection{Mechanical performance of the jaw}

When the device is used to grip a bone piece, the head jaws is normally working under a bending force making the bending strength (or stiffness) important. In order to well determine the mechanical performance of the designed device, both the FEA and bending tests have been carried out to do the analysis numerically and experimentally.

To analyse the mechanical performance of the bio-inspired jaws, it is of necessity to measure the device's maximum gripping force. To achieve this, a piezoresistive thin film force sensor (IMS 0002 C05, I MOTION Inc., China) has been employed. The force applied on the sensor could be obtained through the equation $F=A \cdot R^{B}$, here $R$ is the resistance of the sensor, $\mathrm{A}$ and $\mathrm{B}$ are constants which can be obtained by experiments. To determine A and B, the sensor was mounted onto a hot embossing machine (RYJ-I, developed in our lab) with its end connected with a digital multimeter. A series of constant forces spanning from $220 \mathrm{~N}$ to $300 \mathrm{~N}$ with step size $10 \mathrm{~N}$ were applied on the sensor. The resistances $\mathrm{R}$ can be read from the multimeter. Then the fitting line of this equation was fitted with least square method by Matlab (see Supplementary data-3. Measurement of the maximum gripping force of the head jaws.). The relationship between $F$ and $R$ can be obtained as: $F=178.93 \mathrm{R}^{-0.45}$. To measure the gripping force of the head jaws, five male adults were invited using this device to grip the force sensor, and the resistances shown by the multimeter were recorded accordingly. The resistances were then substituted into the equation mentioned above to obtain the gripping forces F. Results have shown that the average gripping force applied by the head jaws was about $256.3 \mathrm{~N}$.

Two finite element models of the head jaws were established by using the ANSYS software. It has been designed that two identical shapes were adopted in both models; however, one of them was built without the bio-inspired strain-reducing microstructures such that the comparison between two models can be made. To analyze the mechanical performance of the device, a gripping force of $300 \mathrm{~N}$, which is the maximum force that the jaws can apply when an adult male uses the device to grip a bone piece, was used as the load condition during the simulation. Results of the FEA analysis are shown in figure 4a, where 
evident improvement of mechanical performance by using the jaws with the bio-inspired microstructures can be observed. It has been found that the jaw without strain-reducing microstructures generates the maximum bending deformation of $95.3 \mu \mathrm{m}$. In contrast, the maximum bending deformation generated by the jaw with the bio-inspired strain-reducing microstructures is $59.7 \mu \mathrm{m}$, which improves the performance by $37.4 \%$.
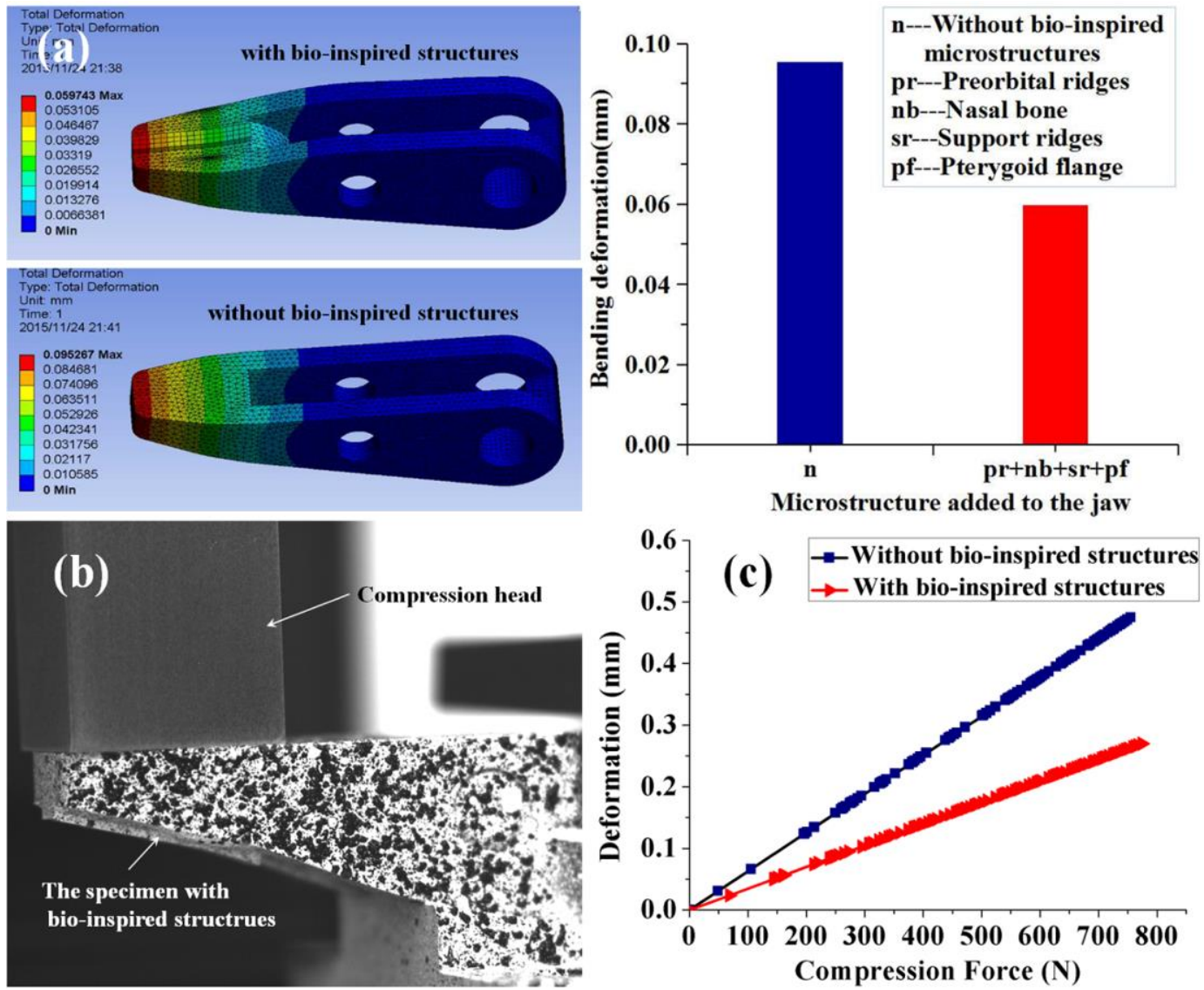

Figure 4. Mechanical performance of the bio-inspired jaw evaluated by using finite element analysis and digital image correlation methods. (a) The comparison between the deformations of two jaws' finite element analysis models which used two identical shapes, but one of them without the bio-inspired strain-reducing microstructures. (b) The digital image correlation technology was used to test the deformation of the jaws. (c) The DIC test results which show the relationships between the applied compression force and the deformations of the specimens.

The bending experiments (figure $4 \mathrm{~b}$ ) were performed by using the digital image correlation (DIC) technology ${ }^{31,32}$ to track and measure the deformation of a jaw with bio-inspired strain-reducing microstructures (hereinafter referred to as specimen A) under a bending moment. A jaw without strain-reducing microstructures (hereinafter referred to as specimen B) was also tested for contrast. The results are shown in figure 4c. It can be found that the deformation of specimen B increases more sharply than that of specimen A as the compression force applied on the specimens increased from $0 \mathrm{~N}$ to $800 \mathrm{~N}$. An evident improvement of mechanical performance by using the jaws with the bio-inspired microstructures has been observed. At a compression force of $300 \mathrm{~N}$ which is approximately the maximum force that the jaws can apply when an adult male uses the device to grip a bone pieces, the deformation of specimen $\mathrm{B}$ is approximately $185.0 \mu \mathrm{m}$. In contrast, the 
deformation of specimen A is about $102.7 \mu \mathrm{m}$ which decreases $44 \%$. The experimental results, which are in accordance with the FEA results, have shown that the bio-inspired strain-reducing structures can dramatically improve the mechanical stiffness of the jaw.

\subsection{Gripping performance of the jaws}

The reliability and security of the gripping are also important for the MIS grasper. In order to validate the gripping performance of the designed jaws, we measured the friction coefficient and the sliding coefficient of the bio-inspired microstructures to the vertebral bone piece. The friction coefficient represents the gripping reliability of the grasper to a bone specimen with flat surfaces; whereas, the sliding coefficient, which is the slope of a slip-line ${ }^{33,34}$, exhibits the gripping reliability to a bone piece with uneven surfaces, and a small one means the occlusal surfaces have better gripping reliability. Specific measurement procedures are represented in the Methods.
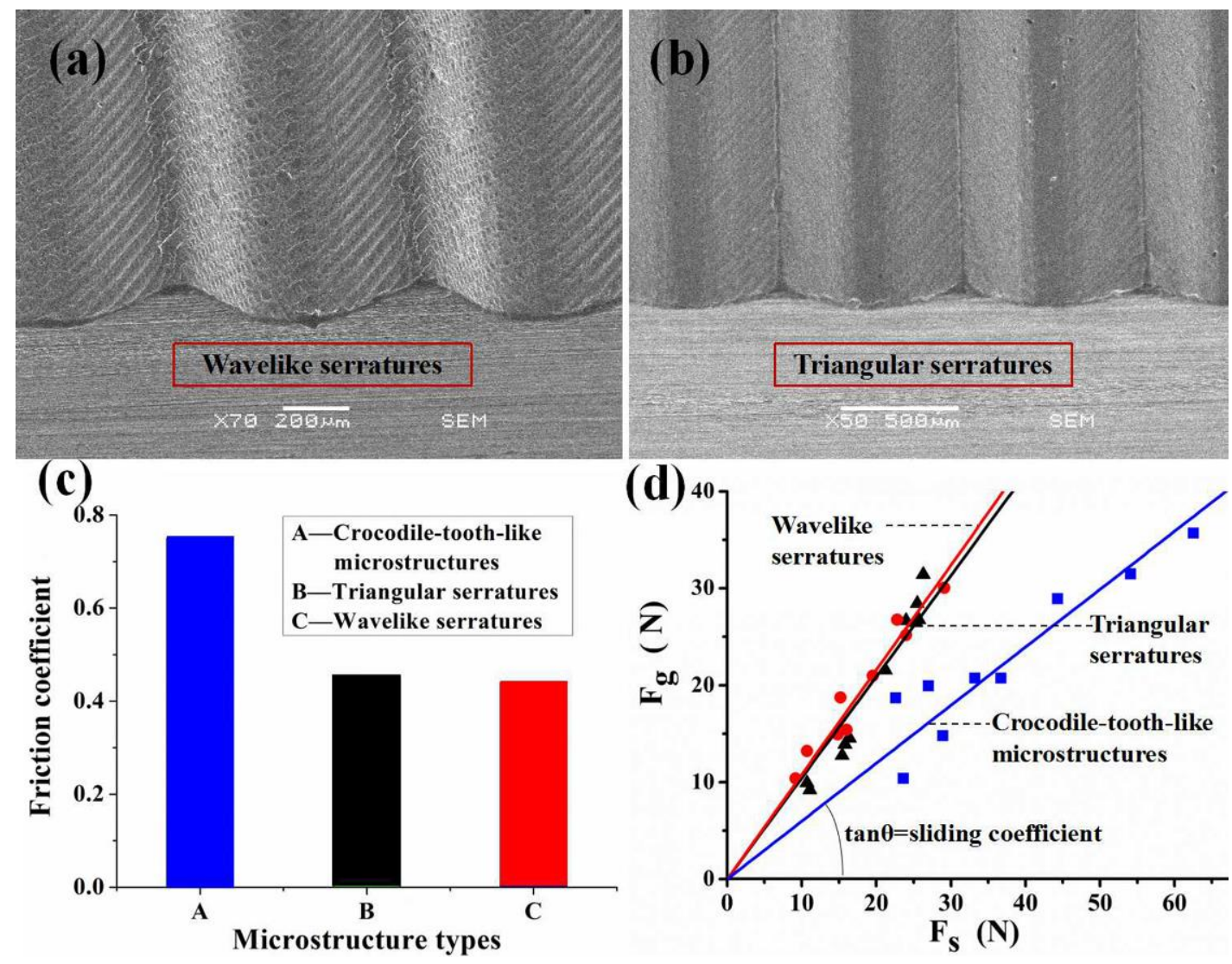

Figure 5 Comparison of the friction coefficients and the sliding coefficients of the crocodile-tooth-like microstructures with the commercial occlusal surfaces. (a) and (b) The commercial wavelike serratures and triangular serratures used to test the friction coefficients and the sliding coefficients. (c) The friction coefficients of the crocodile-tooth-like microstructures, the triangular serratures and the wavelike serratures. (d) The slip-lines of these three types of serratures. The slope of a slip-line is the sliding coefficient.

To effectively assess the performance, two conventional serrated surfaces, i.e. the triangle serratures and the wavelike serratures (figure 5a and 5b) are used as the comparison examples. These two surfaces have been widely used in most commercial MIS graspers. As 
shown in figure $5 \mathrm{c}$, the friction coefficients of crocodile-tooth-like microstructures, triangular serratures and wavelike serratures to the bone are $0.75,0.45$ and 0.44 , respectively. It can be observed that the friction coefficient of the crocodile-tooth-like microstructures to the bone pieces was about 1.70 folds of those of the commercial serrated microstructures. It has been demonstrated that the designed device is able to grab the bone pieces more firmly. In terms of the sliding coefficient, the slip-lines of these three kinds of microstructures are shown in figure 5d. Their sliding coefficients are $0.60,1.06$ and 1.08, respectively, and the sliding coefficient of the crocodile-tooth-like microstructures is approximately $3 / 5$ those of the other two kinds of serratures. In addition, from the figure $5 \mathrm{~d}$, it can be seen that, when a gripping force $\left(\mathrm{F}_{\mathrm{g}}\right)$ of $20 \mathrm{~N}$ is applied to grip a bone piece, a pull force $\left(\mathrm{F}_{\mathrm{s}}\right)$ of $18 \mathrm{~N}-19 \mathrm{~N}$ can pull the specimens to slide on the surfaces of the wavelike serratures and the triangular serratures. In contrast, the bone pieces begins to slide on the surface of the crocodile-tooth-like microstructures until the pull force increases to $33 \mathrm{~N}$. It can be concluded that the jaws with the crocodile-tooth-like microstructures have better reliability in gripping bone pieces. A surgeon could use this grasper to move the bone pieces without sliding.

\subsection{Structure of the MIS device}

The designed MIS device made with 400 stainless steel consists of a handle that can control the opening or closing of the head jaws, a shaft with $110 \mathrm{~mm}$ in length, a grasper head with two jaws used to grip bone pieces and a bolt that could rotate to adjust the angle of the lower jaw (figure 6a). When the head jaws close, this device can be put into the vertebra body through the pedicle canal.

The handle is composed of a movable part and an immovable part. These two parts are connected by a band spring which makes them separated. When the handle is gripped, the head jaws will close. The shapes of the movable and the immovable parts have been both optimized to ensure the convenience and the reliability in surgery operation.

The shaft comprises of two parts with one of them (the lower shaft) connecting the bolt with the lower jaw and transferring the rotation of the bolt to the lower jaw. The other part (the upper shaft) connects the movable part of the handle with upper jaw. The upper jaw and the lower jaw can both rotate around the pin axis at an angle from $0^{\circ}$ to $138^{\circ}$. The bolt is connected with the shaft by the screw making it have the function of slide-lock. As the lower jaw is rotated to an angle, the retreat movement of the shaft will be restricted by the screw, which makes the lower jaw cannot rotate again.

\subsection{Feasibility of resetting vertebral fragments}

We performed in vitro experiments to evaluate the performance of the MIS device. First, a burst fracture was made in a bovine vertebra by using compression. Two $6 \mathrm{~mm}$-diameter pedicle canal were drilled into the vertebra, and the device was put into the vertebral body through the pedicle canal. An endoscope (AnytyWFXS03-45, 3R Eddytek Corp. China) was put into the lumbar through the other canal. The processes of bone pieces resetting could be seen from the display of the endoscope. Because the endoscope contains 4 LED light sources, the bones and the jaws can also be illuminated in the darkness. When undertaking the experiments, the lower head jaw was adjusted to an angle towards a bone piece by turning the adjustment bolt, and the upper head jaw was closed by holding the handle such that a bone 
fragment which invaded into the spinal canal can be gripped. By moving the MIS device, the bone piece can be pulled back to its initial position or out of the vertebral body (figure 6b). To represent the performance of our device, we sprinkled bovine blood on the vertebra to make the bone piece slippery. It was found that the presented MIS device can work well without sliding in gripping the slippery bone piece.

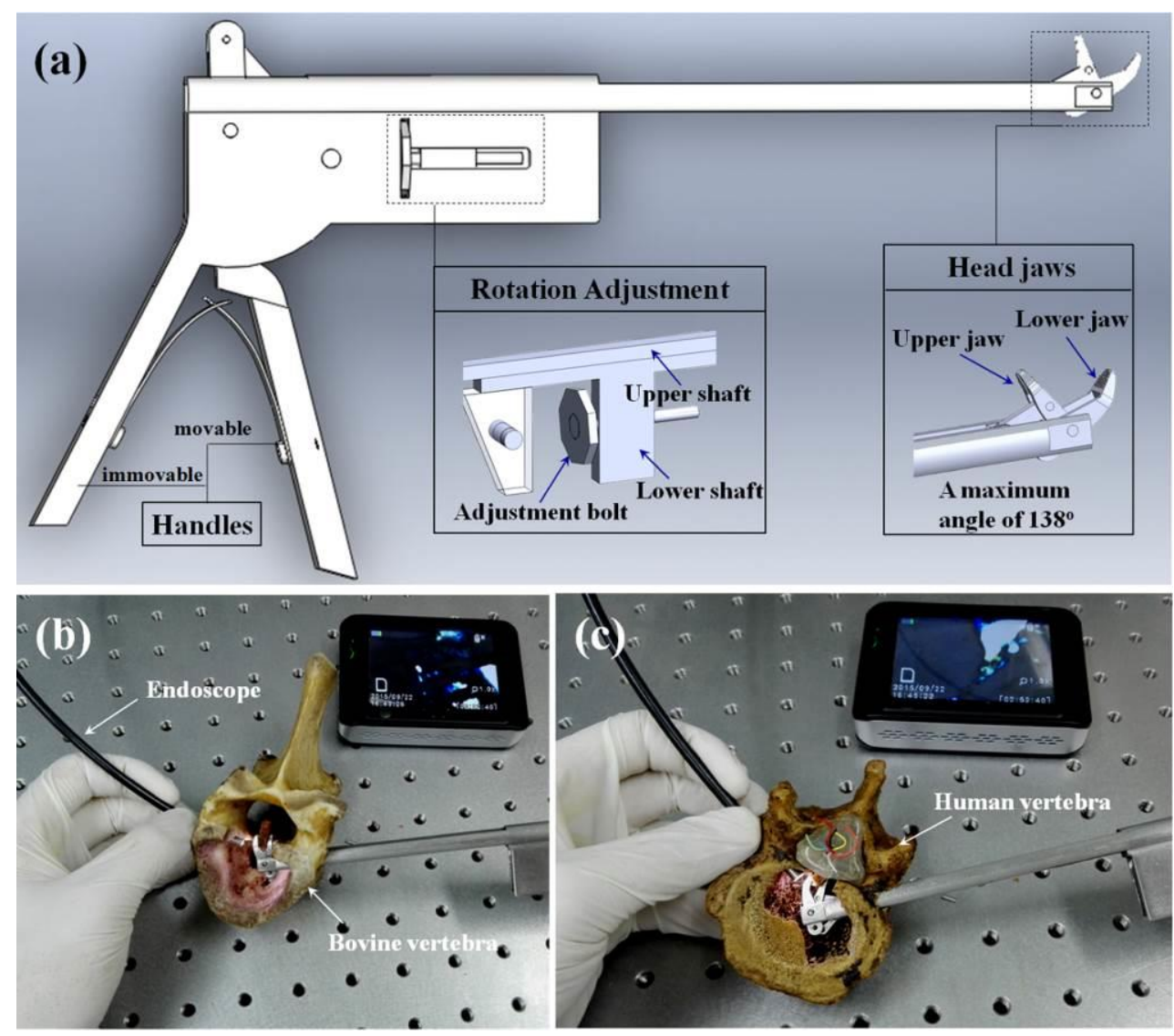

Figure 6. The structure of the minimally invasive surgery device and its feasibility in resetting the bone fragments. (a) The complete structure of the MIS device. (b)Using this MIS device to perform the reset of bone pieces in a burst fractured bovine vertebra. (c) Using this device to reset the bone pieces invading the spinal canal of a burst fractured human vertebra.

To further demonstrate the feasibility of the device, experiments in a human lumbar have also been carried out. First, a nervous cord was manually made by inserting the polydimethylsiloxane (PDMS) into the spinal canal of an L4 lumbar. Then the lumbar was compressed to generate burst fractures (see Methods). Two 6mm-diameter pedicle canals were drilled in the lumbar. Then the device was put into the lumbar body through one canal. An endoscope (AnytyWFXS03-45, 3R Eddytek Corp. Japan) was also put into the lumbar through the other canal to observe the processes of bone pieces resetting. The head jaws of the device were adjusted to aim at a bone fragment embedded into the nervous cord. The adjustment bolt was turned to adjust the lower head jaw to a proper position, and then the upper jaw was closed to grip the bone fragment to pull the fragment out of the nervous cord (figure 6c). During the whole operation process, the device worked flexibly, and the rotate angle of the head jaw $\left(0^{\circ}\right.$ to $\left.138^{\circ}\right)$ can also meet the requirements of gripping bone fragments. 


\section{Conclusions}

Conventional MIS techniques, such as the spinal fusion with screws and bone cements, have limitations in removing of the vertebral pieces invading the spinal canal, which preclude their use in the treatment of lumbar burst fractures with neurologic injury. The novel MIS device presented in this paper can be used with posterior surgical procedures to provide an alternative in resetting the bone fragments. First, this device can be put into the vertebral body through an only $6 \mathrm{~mm}$-diametermini incision. The surgical injuries to the lumbar anatomy, the muscles and the tissues around the vertebras are small. Second, the mechanical performance and the friction coefficient of the jaws with bio-inspired microstructures have been enhanced compared with the commercial graspers. In addition, the sliding coefficient of the crocodile-tooth-like microstructures to bone pieces is only $3 / 5$ those of the commercial ones, which ensures the good performance of the device in gripping and moving the vertebral fragments within the injured vertebral body. More importantly, the maximum open angle of $138^{\circ}$ of the jaws, the slide-lock function and other designs of the device can be efficient in the resetting of the fractured vertebral posterior wall.

It should be noted that further enhancements by using the designed device can also be envisioned. For example, due to the impeding of other bone pieces, it remains difficult to reset a bone fragment. However, because of the designed micro abrasive drill, micro cutting and the grinding tools, this device becomes capable of achieving fracture reduction. These tools can be used firstly to cut off part of the impeding bones, which can decrease the reduction difficulty.

We have demonstrated the feasibility of an MIS device with bio-inspired microstructures in the restoring of a burst fractured spinal vertebra. By mimicking the strain-reducing structures of a crocodilian skull, bending deformation of the device head jaws has dropped $44 \%$ when the bending force of $300 \mathrm{~N}$ was applied. By mimicking the dental form of the crocodilian teeth, the friction coefficient of the jaw's occlusal surface increases 1.70 folds of those of the commercial ones, and the sliding coefficient drops $40 \%$. The presented device has been used to perform the reconstructions of a burst fractured bovine vertebra and a human vertebra in vitro. The results have shown its potentials in providing an alternative to open surgery for the reduction of vertebral burst fractures.

\section{Acknowledgements}

The authors declare that they have no competing financial interests. We thank professor Dianxiu Ning (The First Affiliated Hospital of Dalian Medical University) for helping with CT scanning. This work is supported by the National Natural Science Foundation of China (51475079), the Fundamental Research Funds for the Central Universities (DUT15LAB12). This work is also supported in part by the Science Fund for Creative Research Groups of NSFC (51321004) and National Natural Science Foundation of China (51375076).

\section{References}

[1] Robert G and Harris M 2013 Orthopedic Traumatology (Springer New York) pp 55-68.

[2] Gnanenthiran S, Adie S and Harris I 2012 Nonoperative versus operative treatment for thoracolumbar burst fractures without neurologic deficit: A meta-analysis Clin Orthop Relat $R \mathbf{4 7 0}$ 567-577. 
[3] Wood KB, Buttermann GR, Phukan R, Harrod CC, Mehbod A, Shannon,B, Bono,CM and Harris MB 2015 Operative compared with nonoperative treatment of a thoracolumbar burst fracture without neurological deficit J Bone Joint Surg Am 97 3-9.

[4] Toyone T, et al. 2013 Short-segment fixation without fusion for thoracolumbar burst fractures with neurological deficit can preserve thoracolumbar motion without resulting in post-traumatic disc degeneration: A 10-year follow-up study Spine 38 1482-1490.

[5] Tofuku K, Koga H, Ijiri K, Ishidou Y, Yamamoto T, Zenmyo M, Yone K and Komiya S 2012 Combined posterior and delayed staged mini-open anterior short-segment fusion for thoracolumbar burst fractures J Spinal Disord Tech 25 38-46.

[6] Howard B, Sribnick E and Dhall S 2013 Complete neurologic recovery of spinal cord injury after posterior transpedicular reconstruction for traumatic lumbar burst fracture Spine Journal 13 204-205.

[7] Wu H, Fu C, Yu W and Wang J 2014 The options of the three different surgical approaches for the treatment of Denis type A and B thoracolumbar burst fracture Eur J, Orthop Surg Traumatol 24 29-35.

[8] Boswell S, Sather M, Kebriaei M, Lydiatt K, Bowdino B, Tomes D, Treves J and Hellbusch L 2012 Combined open decompressive laminectomy and vertebroplasty for treatment of thoracolumbar fractures retrospective review of 41 cases Clin Neurol Neurosurg 114 902-906.

[9] Kim M, Eun J and Park J 2011 Radiological and clinical results of laminectomy and posterior stabilization for severe thoracolumbar burst fracture : Surgical technique for one-stage operation $J$ Korean Neurosurg Soc 50 224-230.

[10] Shawky A, Ei-Sabrout E, Ei-Meshtawy M and Boehm H 2014 Management of burst thoracic and thoracolumbar fractures with thoracoscopically assisted anterior corpectomy and posterior short segment percutaneous stabilization The Egyptian Orthopaedic Journal 49 11-17.

[11] Rudolf L 2012 Sacroiliac joint arthrodesis-MIS technique with titanium implants: Report of the first 50 patients and outcomes Open Orthopaedics Journal 6 495-502.

[12] Wood KB, Li WS, Lebl DR, Ploumis A, Wertli MM, Rasmussen-Barr E and Weiser S 2014 Management of thoracolumbar spine fractures Spine Journal 14 145-1641.

[13] Habib N, Maniatis T, Ahmed S, Kilkenny T, Alkaied H, Elsayegh D, Chalhoub M and Harris K 2012 Cement pulmonary embolism after percutaneous vertebroplasty and kyphoplasty: An overview Heart Lung 41 509-511.

[14] Robinson Y and Olerud C 2012 Vertebroplasty and kyphoplasty-A systematic review of cement augmentation techniques for osteoporotic vertebral compression fractures compared to standard medical therapy Maturitas 72 42-49.

[15] Khoo LT, Palmer S, Laich DT and Fessler RG 2002 Minimally invasive percutaneous posterior lumbar interbody fusion Neurosurgery 51 S166-81.

[16] Foley KT, Holly LT and Schwender JD 2003 Minimally invasive lumbar fusion Spine 28 S26-35.

[17] Chung GB, Lee SG, Kim S, Yi BJ and Kim WK 2005 A robot-assisted surgery system for spinal fusion IEEE/RSJ International Conference on Intelligent Robots and Systems 3015-3021.

[18] Lee J, Kim K, Wan KC, Choi S and YS Kim 2008 Human-guided surgical robot system for spinal fusion surgery: CoRASS Robotics and Automation 3881-3887.

[19] Rayfield E, Milner A, Xuan V and Young P 2007 Functional morphology of spinosaur 'crocodile-mimic' dinosaurs J Vertebr Paleontol 27 892-901.

[20] Erickson GM, Gignac PM, Lappin AK, Vliet KA, Brueggen JD and Webb GJW 2014 A 
comparative analysis of ontogenetic bite-force scaling among Crocodylia J Zool 292 48-55.

[21] Mchenry C, Clausen P, Daniel W, Meers M and Pendharkar A 2006 Biomechanics of the rostrum in crocodilians: a comparative analysisusing finite-element modeling Anat Rec Part A 288A 827-849.

[22] Pierce S, Angielczyk K and Rayfield E 2009 Shape and mechanics in thalattosuchian (Crocodylomorpha) skulls: implications for feeding behaviour and niche partitioning $J$ Anat 215 555-576.

[23] Cuff A and Rayfield E 2013 Feeding mechanics in spinosaurid theropods and extantcrocodilians Plos One 8 e65295.

[24] Enax J, Fabritius HO, Rack A, Prymak O, Raabe D and Epple M 2013 Characterization of crocodile teeth: Correlation of composition,microstructure, and hardness J Struct Biol 184 155-163.

[25] Erickson GM, Gignac PM, Steppan SJ, Lappin AK, Vliet KA, Brueggen JD, Inouye BD, Kledzik D and Webb GJW 2012 Insights into the ecology and evolutionary success of crocodilians revealed through bite-force and tooth-pressure experimentation Plos One 7 e31781.

[26] Enax J, Fabritius HO, Rack A, Prymak O, Raabe D and Epple M 2013 Characterization of crocodile teeth: Correlation of composition,microstructure, and hardness J Struct Biol 184 155-163.

[27] Osi A 2014 The evolution of jaw mechanism and dental function in heterodont crocodyliforms Hist Biol 26 279-414.

[28] Kettner M, Schmidt P, Potente S, Ramsthaler F and Schrodt M 2011 Reverse engineering-rapid prototyping of the skull in forensic trauma analysis J Forensic Sci 56 1015-1017.

[29] Platt S, Rainwater TR, Thorbjarnarson JB, Finger AG, Anderson TA and McMurry ST 2009 Size estimation, morphometrics, sex ratio, sexual size dimorphism, and biomass of Morelet's crocodile in northern Belize, Caribbean J Sci 45 80-93.

[30] Bezuijen M, Cox JH, Thorbjarnarson JB, Phothitay C, Hedemark M and Rasphone A 2013 Status of siamese crocodile (crocodylus siamensis) schneider, 1801 (reptilia: crocodylia) in laos J Herpetol 47 41-65.

[31] Pan B 2011 Recent progress in digital image correlation Exp Mech 51 1223-1235.

[32] Bing P and Kai L 2011 A fast digital image correlation method for deformation measurement $O p t$ Lasers Eng 49 841-847.

[33] Westebring-van der Putten E, Hajian M, Goossens R, van den Dobbelsteen J and Jakimowicz J 2010 A laparoscopic grasper handle with integrated augmented tactile feedback, designed for training grasp control Haptics: Generating and Perceiving Tangible SensationsLecture Notes in Computer Science 6192 243-250.

[34] Heijnsdijk E , Visser H, Dankelman J and Gouma D 2004 Slip and damage properties of jaws of laparoscopic graspers Surg Endosc 18 974-979.

[35] Jin L, Phillip P, Edward G, Patricia D and Michael A 2012 Vertebral deformity arising from an accelerated “creep” mechanism Eur Spine J 21 1684-1691.

[36] Sarah D, Jaclyn K, Roderic S and Ray V 2012 Damage mechanics of porcine flexor tendon: mechanical evaluation and modeling Ann Biomed Eng 40 1692-1707. 